\title{
Potential Nephrotoxicity of Lisinopril and Valsartan on Patients with Congestive Heart Failure
}

\author{
Sarini Pani ${ }^{1}$, Melisa I. Barliana², Eli Halimah², Venice Chaeriadi ${ }^{1}$, Mally G. Sholih ${ }^{3}$ \\ ${ }^{1}$ Undata General Hospital, Palu, Central Sulawesi, Indonesia \\ ${ }^{2}$ Department of Pharmacology and Clinical Pharmacy, Faculty of Pharmacy, Universitas Padjadjaran, \\ Jatinangor, West Java, Indonesia. \\ ${ }^{3}$ Department of Obstetrics, Singaperbangsa University, Karawang, West Java, Indonesia
}

\begin{abstract}
Lisinopril (angiotensin converting enzyme inhibitor) and valsartan (angiotensin II receptor blocker) are the first-line treatment for patients with congestive heart failure (CHF). These two drugs potentially cause side effects on renal functions. However, limited information was available regarding the comparison of potential nephrotoxicity of these drugs in Indonesian CHF patients. This research was aimed to compare the potential nephrotoxicity between lisinopril and valsartan in outpatients with CHF at a hospital in Palu, Indonesia. This was an observational study conducted during April-May 2015. Potential nephrotoxicity were assessed by measuring serum creatinin ( $\mathrm{SCr}$ ) and blood urea nitrogen (BUN). Data were obtained from Cardiology Unit from a hospital in Palu, Indonesia. Statistical analysis was conducted using T-test and Mann-Whitney test. The increasing trend of SCr and BUN were observed in lisinopril-treated patients with the mean of increase were $21 \%$ and $59 \%$, respectively. Relatively higher increase was observed in valsartan treatment group with $47 \%$ and $51 \%$ in SCr and BUN, respectively. The analysis showed that there were significant differences in SCr level between lisinopril and valsartan groups $(p=0.001)$, but the opposite results observed in BUN parameter $(\mathrm{p}=0.697)$. Therefore, valsartan was potentially more nephrotoxic than lisinopril based on the increase of SCr parameter. Thus, lisinopril is recommended for CHF patients who are particularly at high risks of having renal impairment.
\end{abstract}

Keywords: lisinopril, valsartan, nephrotoxicity, congestive heart failure

\section{Introduction}

Antihypertensive drugs such as diuretics, angiotensin converting enzyme inhibitors (ACEI), beta blockers, and angiotensin II receptor blockers (ARB) could be used for the treatment of congestive heart failure (CHF). ${ }^{1,2}$ The first-line treatment of $\mathrm{CHF}$ are ACEI or ARB drugs class. ACEI drugs can increase cardiac output and decrease left vetricular filling pressure due to vasodilation effects, while ARB is used as a substitute or adjunctive therapy for ACEI in $\mathrm{CHF}$ treatment. $^{3}$

Corresponding author: Sarini Pani. Undata General Hospital, Palu, Central Sulawesi, Indonesia. Email: sarini.pani@gmail.com. 
Lisinopril may induce nephrotoxicity or acute kidney damage if accompanied by other nephrotoxic drugs. ACEI may exacerbate renal function due to the dominant dilatation of efferent arterioles and consequent reduction in glomerular filtration. Valsartan significantly decreases glomerular filtration rate (GFR), renal plasma flow (RPF) and renal vascular resistance (RVR). ${ }^{4}$

Renal dysfunction is common in patients with $\mathrm{CHF}$ and is associated with high morbidity and mortality. Heart disease and renal dysfunction can worsen each other through various mechanisms such as fluid overload, increased venous pressure, hypoperfusion, neurohormonal activation and inflammation. The interaction between cardiac and renal dysfunction play a major role in disease progression. $^{5}$

The incidences of CHF and chronic kidney disease (CKD) continue to increase with the increasing age of the general population. $\mathrm{CHF}$ and CKD often coexist, which may be associated with common risk factors, such as hypertension, diabetes, and atherosclerosis. Sympathetic activation of the nervous system, renin angiotensin system, inflammation, and oxidative stress are among the mechanism that may worsen those condition. CKD occurs in $30 \%-40 \%$ of patients with $\mathrm{CHF}^{6}$

In Indonesia, the highest prevalence of coronary heart disease was observed in Central Sulawesi $(0.8 \%)$ followed by North Sulawesi (0.7\%), DKI Jakarta (0.7\%), and Aceh $(0.7 \%))^{7}$ Information regarding comparison of potential nephrotoxicity of lisinopril and valsartan in this population was limited.

Therefore, this research was conducted to assess potential nephrotoxicity between lisinopril and valsartan in CHF patients at one hospital in Palu, Central Sulawesi, Indonesia.

\section{Methods}

This was an observational study conducted in two months during April-May 2015. We

Table 1. Characteristics of subjects

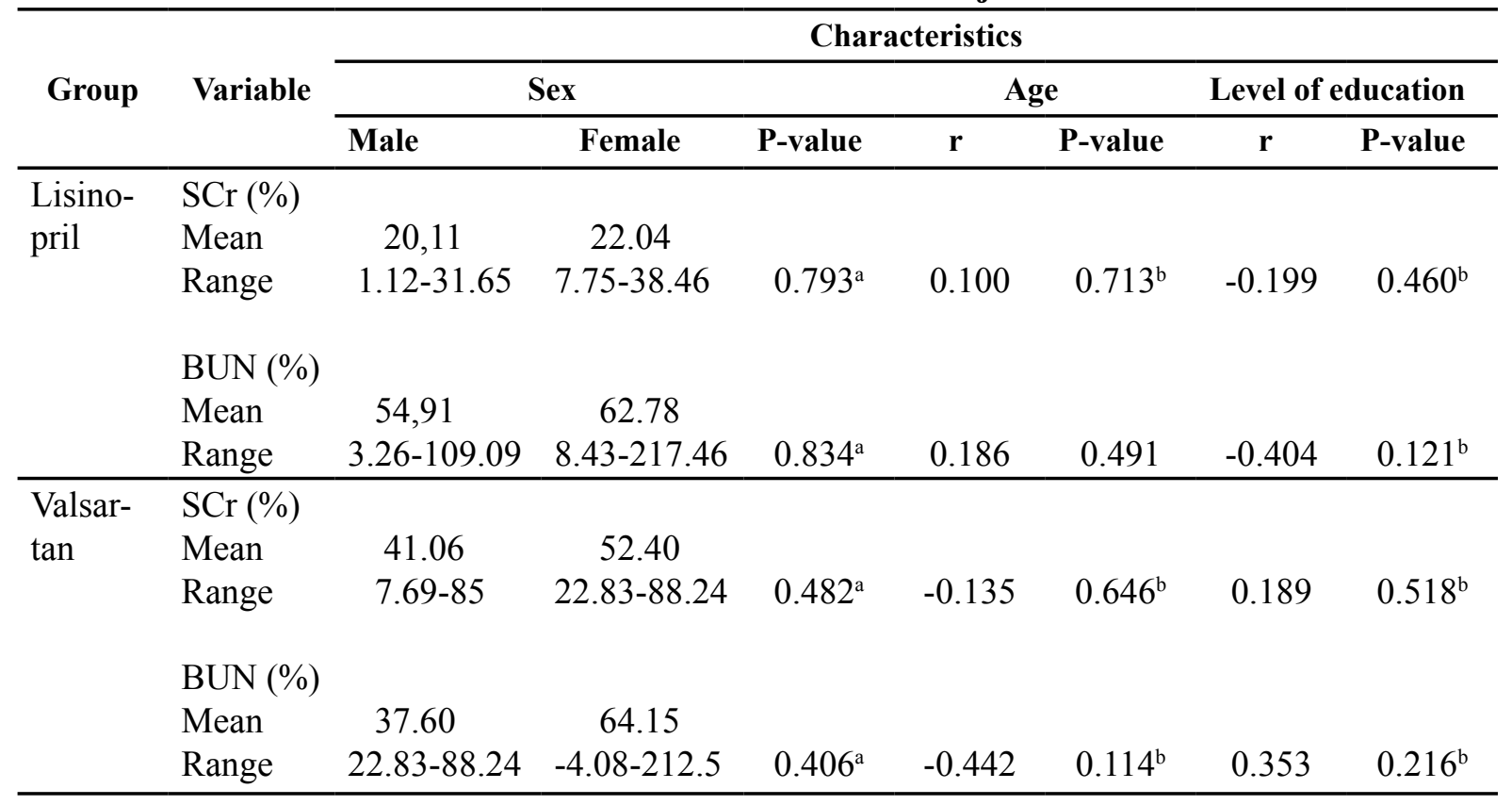

a) Mann-Whitney test; b) Spearman's test 
included thirty subjects aged $\geq 18$ years with hypertension, CHF, using lisinopril $5 \mathrm{mg}$ or valsartan $80 \mathrm{mg}$, with normal serum creatinine (SCr) and blood urea nitrogen (BUN) levels $(0.6-1.3 \mathrm{mg} / \mathrm{dl})$ at initial period of study. The conduct of this research was approved by ethical committee Universitas Padjadjaran.

Potential nephrotoxicity was assessed by evaluating $\mathrm{SCr}$ and BUN levels during initial and the end of study. Baseline characteristics based on sex, age, and level of education of two groups were compared. Statistical analysis was performed using using Mann-Whitney, Spearman, and coefficient correlation test. The data were obtained from cardiology outpatient clinic at Undata Hospital, Palu, Indonesia.

\section{Results and Discussion}

The subjects were grouped as follows: 16 lisinopril-treated and 14 valsartan-treated. We found out that there were no significant differences on the baseline of those two groups $(p>0.05)$ (Table 1). Therefore, there were no significant influences from sex, age and level of education on renal functions.

The increasing trend of $\mathrm{SCr}$ and BUN were observed in lisinopril-treated patients with the mean of increase were $21 \%$ and $59 \%$, respectively. Relatively higher increase was observed in valsartan treatment group with $47 \%$ and $51 \%$ in SCr and BUN, respectively. The analysis showed that there were significant differences in $\mathrm{SCr}$ level between lisinopril and valsartan groups $(\mathrm{p}=0.001)$, but the opposite results observed in BUN parameter $(\mathrm{p}=0.697)$. Therefore, valsartan was potentially more nephrotoxic than lisinopril based on the increase of SCr parameter (Table 2).

Valsartan causes nephrotoxicity by decreasing glomerular capillary hydrostatic pressure which can ultimately decrease glomerular ultrafiltration. This occurs when the regulation of blood flow is reduced in the glomerular afferent artery and the efferent artery undergoes vasconstriction in maintaining the glomerular capillary hydrostatic pressure for ultrafiltration. ${ }^{8}$ Lisinopril and valsartan may reduce intrarenal perfusion and decrease intracapillary hydraulic pressure thereby reducing the filtration fraction and finally GFR. ${ }^{9}$

According to De Broe et al., inhibition of renin angiotensin aldosteron system (RAAS) by lisinopril and valsartan may also weaken renal fibrosis and decrease excretion of fibrogenic cytokines. ${ }^{10}$ The ACEI group may increase SCr by reducing $30 \%$ of efferent vascular tone to reduce the pressure of glomerular filtration. However, it can lead to diuresis which can

Tabel 2. SCr and BUN levels in both groups

\begin{tabular}{lccc}
\hline \multirow{2}{*}{ Variable } & \multicolumn{2}{c}{ Treatment } & \multirow{2}{*}{ P-value } \\
\cline { 2 - 3 } & $\begin{array}{c}\text { Lisinopril 5 } \mathbf{~ m g} \\
(\mathbf{n = 1 6 )}\end{array}$ & $\begin{array}{c}\text { Valsartan 80 } \mathbf{~ m g} \\
(\mathbf{n = 1 4})\end{array}$ & $0,001^{\mathrm{a} *}$ \\
\hline $\mathrm{SCr}(\mathrm{mg} / \mathrm{dl}):$ & $21.1(10.7)$ & $46.7(24.0)$ & \\
Mean (SD) & $1.1-38.5$ & $7.7-88.2$ & \\
Range & & & $0,697^{\mathrm{b}}$ \\
& & $50.9(51.9)$ & \\
BUN (mg/dl) : & $58.8(536)$ & $-4.08-212.5$ & \\
Mean (SD) & $3.26-217.46$ & & \\
Range & &
\end{tabular}


increase $\mathrm{SCr}$ and blood pressure that may contribute to kidney dysfunction. ${ }^{11}$

The increase of $\mathrm{SCr}$ in the valsartan group was higher, which indicates that valsartan may have higher risk on renal function. This finding was similar with other study showing that valsartan caused significant decrease in GFR and RPF without full recovery of renal function. It also resulted in decreased renal vascular resistance (RVR) and tubular sodium loss. ${ }^{12-14}$ Regarding efficacy of both drugs, previous study indicated that evidence does not support a meaningful difference between ACEI and ARBs for any blood pressure outcome except medication side effects. ${ }^{15}$

Valsartan or lisinopril are often used in combination with other drugs such as other diuretics and non-steroid anti-inflammatory drugs. In this study, selection of subject (normal $\mathrm{SCr}$ and BUN value) was based on the presence of valsartan or lisinopril, regardless the concomitant medications used, therefore the possibility of selection bias could be excluded.

\section{Conclusion}

Based on the comparison of potential nephrotoxicity between lisinopril and valsartan in CHF patients, valsartan was potentially more nephrotoxic than lisinopril based on the increase of $\mathrm{SCr}$ value. Thus, lisinopril is recommended for CHF patients with high risk of renal impairment.

\section{Acknowledgement}

The authors acknowledge the medical staff of Undata Hospital, Palu, Indonesia, for their assistance and participation during the study.

\section{Funding}

This research was not funded by any source of grants.

\section{Conflict of Interest}

The authors declared no potential conflicts of interest with respect to the research, authorship, and/or publication of this article.

\section{References}

1. Thomas R, Kanso A, Sedor JR. Chronic kidney disease and its complications. Primary Care. 2008;35(2):329-337.

2. Jessup M, Abraham WT, Casey DE, Feldman AM, Francis GS, Ganiats TG et al. ACCF/AHA Guidelines for the diagnosis and management of heart failure in Adults. ACCF/AHA. Journal of American College of Cardiology. 2009;119:16.

3. Philip J, Demers C, Robert S, Peter P, Liu MD. Angiotensin receptor blockers in heart failure: meta-analysis of randomized controlled trials. Journal of the American College of Cardiology. 2012;39(3):463464.

4. Onuigbo MA, Agbasi N. Quadruple whammy- A preventable newly described syndrome of post-operative AKI in CKD II and CKD III patients. Nigerian Journal of Clinical Practice. 2014; 17(5) : 649654

5. Metra M, Cotter G, Gheoghiade M, Cas LD, Voors A. The role of the kidney in heart failure. European Heart Journal. 2012; 33: 2135-2143

6. Udani SM, Koyner JL. The effects of heart failure on renal function. Cardiology Clinics. 2010;28(3):453-465.

7. Hill NR, Fatoba ST, Oke JL. Global prevalence of chronic kidney disease -a systematic review and meta-Analysis. PLoS ONE. 2016;11(7):e0158765.

8. Prodjosudjadi W, Suhardjono A. Endstage renal diseases in Indonesia: treatment development. Ethnicity and Diseases. 2009;19(1):333-36.

9. Galie N. New horizons in antihypertensive therapy:gopten (trandolapril). European 
Respiratory Review. 2013; 22(130):503514

10. Charles F, Lacy, Lora L, Amstrong M. Drug Information Handbook, $17^{\text {th }}$ Edition. 2009. American Pharmacists Association: United States.

11. Maeder MT, Rickli H, Pristerer ME. Incidence, clinical predictors, and prognostic impact of worsening renal function in elderly patients with chronic heart failure on intensive medical therapy. American Heart Journal. 2012;163(3):407-414.

12. Dusing R. Pharmacological interventions into the renin-angiotensin system with ACE inhibitors and angiotensin II receptor antagonists: effects beyond blood pressure lowering. Therapeutic Advances in Cardiovascular Diseases. 2016;10(3):151-161.
13. Perazella MA. Renal vulnerability to drug toxicity, section of nephrology. Clinical Journal of American Society of Nephrology. 2009;4:1275-1276.

14. John JV, Murray MC, Adamopoulos S, Anker SD. Guidelines for the diagnosis and treatment of acute and chronic heart failure. European Heart Journal. 2012;33:17-94

15. Powers BJ, Coeytaux RR, Dolor RJ, et al. Updated report on comparative effectiveness of ACE inhibitors, ARBs, and direct renin inhibitors for patients with essential hypertension: much more data, little new information. Journal of General Internal Medicine. 2012;27(6):716-729. 